\title{
A NOTE ON THE INTEGRAL EQUATION METHODS IN POTENTIAL THEORY*
}

By M. MAITI (Institute of Theoretical and Applied Mechanics, University of Kentucky)

Abstract. This note defines the kernel functions for the generation of conjugate harmonic functions in the theory of singular integral equations adapted to the boundary value problems of potential theory and elastostatics.

1. Introduction. Jaswon [1] has recently introduced a new approach to solve the boundary value problems of potential theory in terms of integral equations. These techniques have been proved to be successful both in potential theory [2] and in elastostatics [3]. This note explains the kernel function $\theta$, which generates the harmonic conjugates, because difficulties are generally encountered in practice with Jaswon's definition. The main objective here is to define the function $\theta$ properly so that the physical problems can conveniently be formulated in terms of it. Jaswon's symbolism and conventions will be used throughout this paper and it is unnecessary to explain them again.

2. Simple layer potential. Let $L$ be a single, simple, smooth contour bounding a domain $D$. We may express a harmonic function $\phi$ in $D$ as

$$
\phi(\mathbf{P})=\int_{L} \log |\mathbf{P}-\mathbf{q}| \sigma(\mathbf{q}) d q,
$$

where $\mathbf{P}, \mathbf{q}$ are two vector variables specifying field point in $D$ and source point on $L$ respectively and where $d q$ is the arc differential at $\mathbf{q} ; \sigma(\mathbf{q})$ is the density function defined on $L$. As the field point $\mathbf{P}$ approaches a point $\mathrm{p}$ on $L$ itself, the simple layer potential remains continuous, so that we may write

$$
\phi(\mathbf{p})=\int_{L} \log |\mathbf{p}-\mathbf{q}| \sigma(\mathbf{q}) d q,
$$

where the above integral will be interpreted as Cauchy's principal value. Given $\phi(p)$ on $L,(2)$ reduces to Fredholm's integral equation of first kind which can be solved uniquely for $\sigma$ except on certain contours [1]. This solution for $\sigma$ generates an interior harmonic function

$$
\phi_{i} \equiv \phi_{i}(\mathbf{P})=\int_{L} \log |\mathbf{P}-\mathbf{q}| \sigma(\mathbf{q}) d q ; \quad \mathbf{P} \subset D,
$$

and an exterior harmonic function

$$
\phi_{\mathbf{c}} \equiv \phi_{\mathbf{c}}(\mathbf{P})=\int_{L} \log |\mathbf{P}-\mathbf{q}| \sigma(\mathbf{q}) d q ; \quad \mathbf{P} \subset D .
$$

each of which reduces to $\phi(\mathrm{p})$ on $L$. Also we have that

$$
\phi_{i}^{\prime}+\phi_{0}^{\prime}=2 \pi \sigma,
$$

*Received January 27, 1967. 
where ()$^{\prime}$ denotes the normal derivative into the respective domains. It is interesting to note that the same density function $\sigma$ generates complex conjugate

$$
\bar{\phi}(\mathbf{P})=\int_{L} \theta(\mathbf{P}-\mathbf{q}) \sigma(\mathbf{q}) d q,
$$

where $\theta(\mathbf{P}-\mathbf{q})$ is the complex conjugate of $\log |\mathbf{P}-\mathbf{q}|$. The following definition of $\theta$ holds for all positions of $\mathbf{P}$, whether in $D$ or outside $D$ or on $L$, and some interior functions will be considered for illustrations.

3. Definition of $\theta$. Let $\mathrm{OA}$ be a fixed line (Fig. 1), which we may term the 'base line'. The potential $\theta$, which is contributed at $\mathbf{P}$ by a source at $\mathbf{q}$, is measured between the vector $\mathbf{P}-\mathbf{q}$ and the fixed direction OA. For a continuous distribution of source on $L$ we adopt the following procedure. Let the source point $q$ coincide first with the point 0 of the base line; now vary $\theta$ continuously from this starting value when $q$ completes a circuit moving along the range of integration on $L$. This implies that $\theta$ suffers a jump at its starting value and also that $\theta$ can exceed $2 \pi$ but reverts to its starting value on completing a circuit of $L$, multivaluedness being thereby eliminated. The base line is important in that $\theta$ must always be measured starting from this line whatever be the position of the field point; thus keeping the direction $O A$ and the base line fixed, the 'branch' of the function $\theta$ should be changed with the variation of the field point $P$. The same definition holds also when $\mathbf{P}$ lies on $L$, i.e. when $\mathbf{P}=\mathbf{p}$, except that $\theta$ suffers another jump of amount $\pi$ when $\mathbf{q}$ crosses $\mathbf{p}$. Thus, in this case, $\theta$ suffers two jumps, one at $\mathbf{p}$ and the other at the starting value.

4. Illustrations. Let us consider a circular domain $D$ (Fig. 2) with radius ' $a$ ' and center at $\mathbf{C}$. If there exists a harmonic function $\phi=$ constant in $D$, its harmonic conjugate should also be constant in $D$. It will be shown here that this is true for the above definition of $\theta$. Let $O A$ be the base line and $\mathrm{P}$ be any other point on the diameter $A^{\prime} O$. For convenience, let us denote the angle which contributes to the potential at $\mathbf{P}$ by $\theta$, and that which contributes to the potential at $\mathbf{C}$ by $\omega$, i.e.,

$$
\bar{\phi}(\mathbf{P})=\int \theta(\mathbf{P}-\mathbf{q}) d q, \quad \bar{\phi}(\mathbf{C})=\int \omega(\mathbf{C}-\mathbf{q}) d q,
$$

where $\sigma=1$ (assumed without loss of generality), and $\theta, \omega$ are measured continuously from their starting values at $O A$. Then from (7) we obtain

$$
\bar{\phi}(\mathbf{P})-\bar{\phi}(\mathbf{C})=\int(\theta-\omega) d q .
$$

Consider now two points $q$ and $\mathbf{q}^{\prime}$ on $D$, which are image points with respect to the line $A^{\prime} O$ and define $\theta, \omega$ at these points as shown in Fig. 2. It is obvious that $\theta-\omega<0$ at $q$ and $\theta-\omega>0$ at $\mathbf{q}^{\prime}$, and also that

$$
|\theta-\omega|_{\mathbf{q}}=|\theta-\omega|_{\mathbf{q}^{\prime}},
$$

where $|\theta-\omega|_{\mathbb{q}}$ means that $|\theta-\omega|$ is evaluated at $\mathbf{q}$ etc. Hence the integral in (8) vanishes by symmetry, and we get

$$
\Phi(\mathbf{P})=\bar{\phi}(\mathbf{C}) .
$$

The above result shows that $\bar{\phi}(\mathbf{P})$ remains constant with our definition of $\theta$ and

for all $\mathbf{P}$ in $D$.

$$
\Phi(\mathbf{P})=\Phi(\mathbf{C})=a \int_{0}^{2 \pi}(\pi+\theta) d \theta=4 a \pi^{2}
$$


Let us consider the case when the field point is on $D$. The coordinate system is chosen in such a way that the $x$-axis coincides with the base line and $p$ is such that it makes an angle $\alpha$ with the $x$-axis (Fig. 3). Divide the boundary into two parts $A B$ and BCEFA. If $\mathrm{q}$ is any point on $A B$ making an angle $\eta$ with $x$-axis, then $\theta$ is given by

$$
\theta(\mathbf{p}-\mathbf{q})=\frac{1}{2}[\pi+\alpha+\eta],
$$

so that the contribution to $\Phi$ from the arc $A B$ is

$$
\frac{1}{2} a \int_{0}^{\alpha}(\pi+\alpha+\eta) d \eta=\frac{1}{2} a\left[\pi \alpha+\frac{3 \alpha^{2}}{2}\right] .
$$

If $\mathrm{q}$ is anywhere on BCEFA making an angle $\eta$ with $x$-axis, then $\theta$ is given by

$$
\theta(\mathbf{p}-\mathbf{q})=\frac{1}{2}[3 \pi+\alpha+\eta]
$$

so the contribution to $\Phi$ from the arc BCEFA is

$$
\frac{1}{2} a \int_{\alpha}^{2 \pi}(3 \pi+\alpha+\eta) d \eta=\frac{1}{2} a\left[8 \pi^{2}-\pi \alpha-\frac{3 \alpha^{2}}{2}\right] .
$$

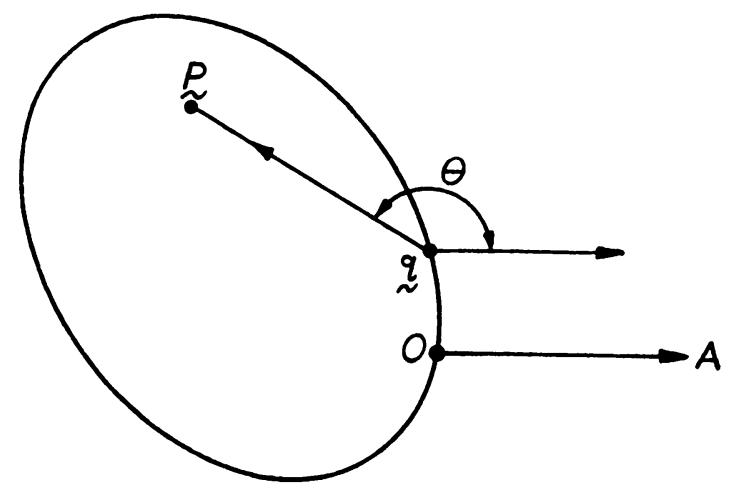

Figure 1

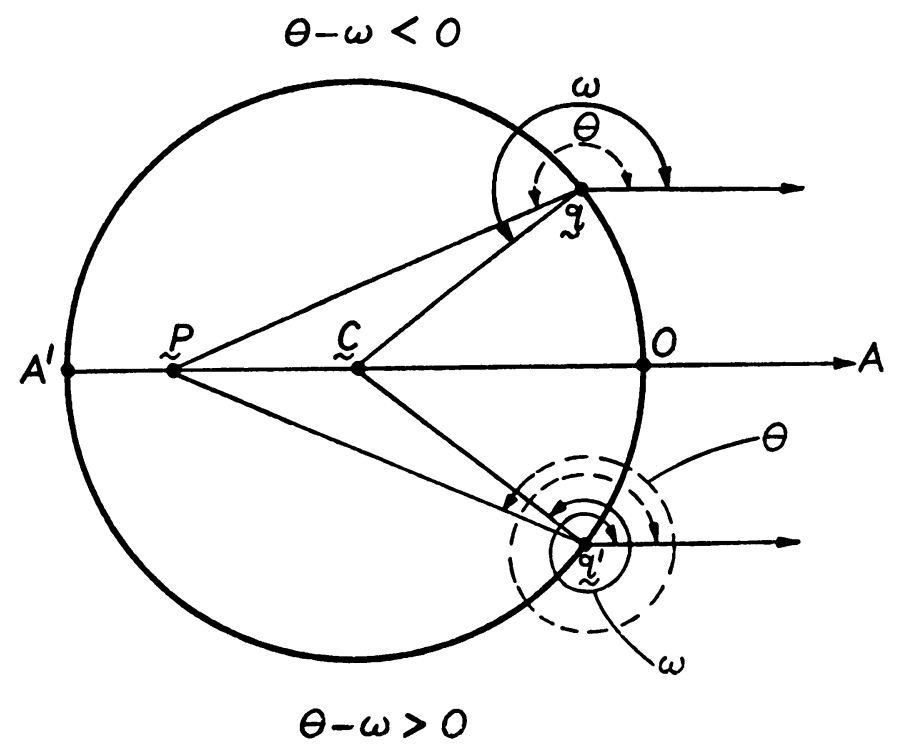

FIGURE 2 


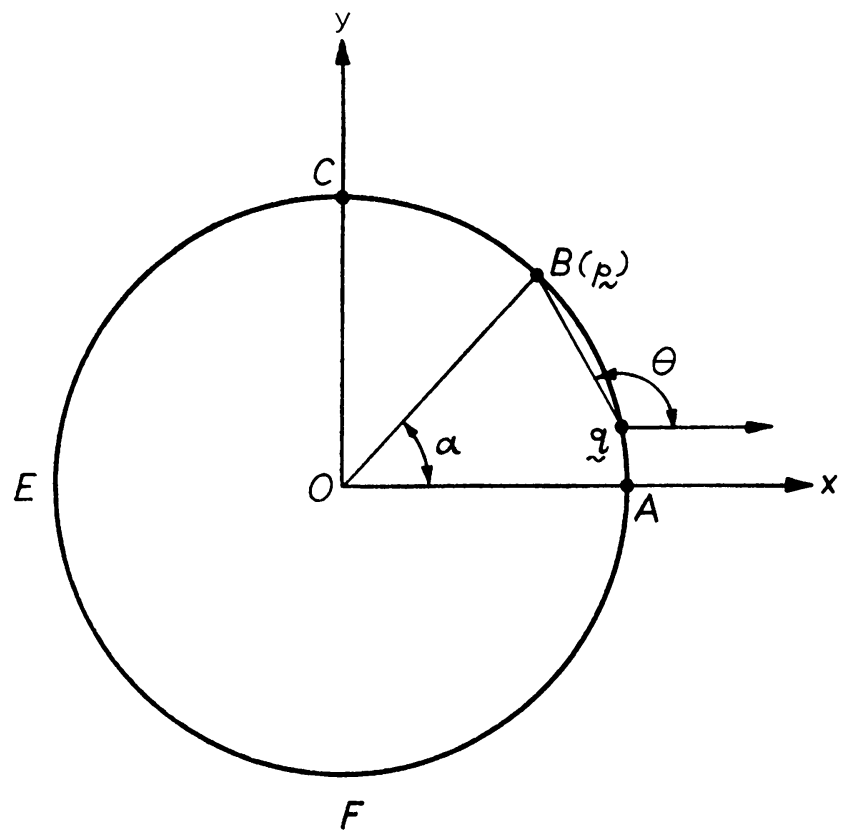

Figure 3

Adding (12 and (14) we obtain

$$
\Phi(\mathbf{p})=4 a \pi^{2}, \text { which agrees with }(10) .
$$

Thus, with our definition of $\theta$, we are able to show that $\bar{\phi}$ remains constant everywhere in and on $D$.

Consider now a more rapidly varying trial function $\phi_{i}=x$, instead of being a constant, in which case $\phi_{e}=a^{2} \cos \eta / r$, so that from (5) it follows that

$$
\sigma=(1 / 2 \pi)\left[\phi_{i}^{\prime}+\phi_{e}^{\prime}\right]=-\cos \eta / \pi,
$$

where $(r, \eta)$ are the polar coordinates. Thus for the above $\mathbf{p}, \phi(\mathbf{p})=a \cos \alpha$ and $\sigma$ must generate $\phi(\mathbf{p})=y \equiv a$ sin $\alpha$, apart from an arbitrary constant. Let $\phi(\mathbf{p})=\bar{\phi}_{1}(\mathbf{p})+$ $\bar{\phi}_{2}(\mathbf{p})$, where $\bar{\phi}_{1}(\mathbf{p})$ arises from the arc $A B$ and $\phi_{2}(\mathbf{p})$ arises from the arc BCEFA. Thus from (11), (13) and (15) it follows that

$$
\begin{aligned}
\bar{\phi}_{1}(\mathbf{p}) & =-\frac{a}{2 \pi} \int_{0}^{\alpha}(\pi+\alpha+\eta) \cos \eta d \eta \\
& =-\frac{a}{2 \pi}[\pi \sin \alpha+2 \alpha \sin \alpha+\cos \alpha-1], \\
\bar{\phi}_{2}(\mathbf{p}) & =-\frac{a}{2 \pi} \int_{\alpha}^{2 \pi}(3 \pi+\alpha+\eta) \cos \eta d \eta \\
& =\frac{a}{2 \pi}[3 \pi \sin \alpha+2 \alpha \sin \alpha+\cos \alpha-1],
\end{aligned}
$$

whence $\Phi(\mathbf{p})=\Phi_{1}(\mathbf{p})+\bar{\phi}_{2}(\mathbf{p})=a \sin \alpha \equiv y$. These are useful checks on the definition of $\theta$. For the boundaries other than a circle we must adopt a numerical procedure. 
5. Conclusions. The important fact emerges from the above definition that $\int \theta(\mathbf{p}-\mathbf{q}) d q$ never vanishes, which implies that the solution of (6) for $\sigma$ is unique on any contour $L$. This is an advantage over (2) which fails to give solutions for $\sigma$ on $\Gamma$-contour [1].

\section{REFERENCES}

[1] M. A. Jaswon, Integral equation methods in potential theory. I, Proc. Roy. Soc., A, 275 23-32 (1963)

[2] G. T. Symm, Integral equation methods in potential theory. II, Proc. Roy. Soc., A, 275 33-46 (1963)

[3] M. A. Jaswon, M. Maiti and G. T. Symm, Numerical biharmonic analysis and some applications, International Journal of Solids and Structures 3, 309 (1967) 